\title{
Plataformas digitais e departamentos de comunicação/relações públicas: uma revisão sistemática
}

\author{
Allana Albuquerque ${ }^{1}$, Telmo Silva ${ }^{1}$ \\ allana.cs@gmail.com; tsilva@ua.pt \\ ${ }^{1}$ Digimedia, Departamento de Comunicação e Arte, Universidade de Aveiro, Universidade de Aveiro, \\ Campus Universitário de Santiago 3810-193, Aveiro, Portugal
}

DOI: 10.17013/risti.42.62-77

\begin{abstract}
Resumo: Este artigo apresenta estudos, correntes teóricas e autores que fazem parte do atual estado da arte referente à comunicação/relações públicas e plataformas digitais. Em uma revisão sistemática, usando a metodologia Prisma, efetua o levantamento de artigos acadêmicos, publicados no período de 2015 a 2020, oriundos de dois bancos de dados: Web of Science e Scopus. Como resultado quantitativo, observa-se que os estudos relacionados a departamentos de comunicação não representam nem $1 \%$ do total de pesquisas relacionadas a plataformas digitais, tecnologias digitais ou sistemas de informação. Os resultados abrangeram a discussão sobre o conceito de plataformas digitais; a transformação digital dos departamentos de comunicação, e o papel das mídias sociais. Demonstram ainda a predominância de estudos relacionados à estratégia de comunicação nas mídias sociais, com foco na relação entre organização e seus públicos. Novas pesquisas devem explorar o gerenciamento das informações produzidas com o uso de plataformas digitais.
\end{abstract}

Palavras-chave: departamentos de comunicação social; departamento de relações públicas; plataformas digitais; mídias sociais; transformação digital.

\section{Digital platforms and communication/public relations departments: A systematic literature review}

Abstract: This article presents studies, theoretical currents and authors that are part of the current state of the art regarding communication / public relations and digital platforms. In a systematic review, using the Prisma methodology, it carries out a survey of academic articles, published in the period from 2015 to 2020, from two databases: Web of Science and Scopus. As a quantitative result, it is observed that studies related to communication departments do not represent even $1 \%$ of the total research related to digital platforms, technologies or information systems. The results covered the discussion on the concept of digital platforms, the digital transformation of communications departments, and the role of social media. They also demonstrate the predominance of studies related to the communication strategy in social media, focusing on the relationship between organizations and 
their audiences. New research should explore the management of information produced using digital platforms.

Keywords: communication departments; public relations departments; digital platforms; social media; digital transformation.

\section{Introdução}

Na chamada Sociedade em Rede, recairá em todas as organizações e seus setores, inclusive nos departamentos de comunicação, a transformação digital ao ser entendida como "o processo que orienta a cultura, a estratégia, as metodologias e as capacidades de uma organização a partir das tecnologias digitais" (Cuenca-Fontbona, Matilla, \& Compte-Pujol, 2020, p. 78). O objetivo da pesquisa é apresentar estudos, correntes teóricas e autores que fazem parte do atual estado da arte referente aos constructos comunicação e plataformas digitais (PD), mais especificamente à transformação digital dos departamentos de comunicação social/relações públicas. Os resultados apresentados buscam responder à seguinte questão de investigação: como se relacionam os estudos sobre plataformas digitais e departamentos de comunicação social?

Nesses setores circulam informações, cujos conteúdos são formatados para atingirem diversos públicos através de ferramentas tecnológicas. As PD dão às instituições o poder de alcançar milhares de pessoas, sem necessitarem do apoio da imprensa ou de terem de investir grande quantidade de recursos financeiros em publicidade. As organizações passam a conversar diretamente com seus clientes/públicos. As PDs passam a permitir uma interação mais direta e uma avaliação mais fácil dos resultados dos esforços realizados por meio de investimentos em comunicação.

A primeira parte deste artigo é destinada à descrição da estratégia de busca dos trabalhos selecionados. Essa etapa apresenta autores a partir dos quais se definiram as palavraschave, o processo de seleção e os principais autores identificados, bem como uma análise quantitativa dos trabalhos identificados e das palavras-chave usadas. Nas duas bases de dados renomadas, utilizaram-se termos em inglês e português, em suas formas singular e plural. Essa descrição permite a continuidade do acompanhamento da temática por pesquisadores interessados sobre o tema. A segunda parte à categorização e discussão dos principais estudos selecionados. A partir desses estudos buscou-se identificar: como são tratados os conceitos de plataformas digitais e departamentos de comunicação e como eles se relacionam. Tais estudos foram abordados em três categorias: conceito de plataformas digitais; a transformação digital dos departamentos de comunicação, e o papel das mídias sociais.

\section{Metodologia}

A metodologia utilizada foi baseada nas diretrizes Prisma: identificação, seleção, elegibilidade e inclusão (Moher et al., 2009). A partir da análise de revisões e artigos acadêmicos, publicados no período de 2015 a 2020, oriundos de dois bancos de dados: Web of Science (W) e Scopus (S). O primeiro reúne mais de 12 mil periódicos de impacto mundial, sendo o segundo o maior banco de dados de resumos e citações da literatura revisada por pares (Soriano, Alvarez, \& Valdes, 2018). 
Com base no objetivo proposto, foram utilizadas as seguintes palavras-chave principais para as pesquisas: "digital platform" ${ }^{*}$ " "digital technolog * OR "information system*" relacionados com dois termos secundários: "public relation" e "communication*". O primeiro conjunto de termos principais escolhidos decorre dos estudos de Reuver et al. (2018) e Passarelli, Ribeiro, Oliveira e Mealha (2014). Esses termos são usados como sinônimos de sistemas que utilizam a Web como suporte de interação entre os usuários, embora o termo plataforma digital substitua o emprego da expressão tecnologia da informação e comunicação, e absorva "o conceito de sistema tecnológico ou infraestrutura tecnológica” (Passarelli et al., 2014, p. 2160). Os termos secundários escolhidos se demonstraram adequados, em virtude do baixo quantitativo de estudos que relacionam as duas temáticas.

\section{Resultados}

Uma busca inicial (Wo) foi feita na categoria "administração pública” no banco de dados Web of Science (W) para identificar como o assunto tem sido abordado nessa área. Dos mais de 60 mil estudos indexados, no entanto, identificaram-se apenas nove com public relation* ou communication*, no resumo, palavras-chave ou título na categoria de periódicos da administração pública. Na base Scopus (So), dos mais de 90 mil estudos indexados, 14 continham public relation* ou communication*, nas categorias "Computer Science", "Social Sciences" e "Multidisciplinary".

Nesta primeira rodada de buscas, dos 23 inicialmente selecionados a partir das duas bases de dados, permaneceram 12 trabalhos após a exclusão daqueles pertencentes a periódicos relacionados às áreas de agricultura, gerontologia, ciência da saúde, planejamento urbano, engenharia elétrica e civil, até mesmo de administração quando o tema estava muito fora do procurado. Essa primeira busca já sinalizou para a escassez de pesquisas relacionadas ao conceito de plataformas digitais e comunicação.

Em uma outra rodada de pesquisa, foram utilizados três conjuntos de palavras-chaves mais específicas, representando, respectivamente, os conjuntos $\mathrm{W}_{1}$, W2 e W3 na base de dados Web of Science e os conjuntos S1, S2 e S3 na Scopus: (i) public relations OR communication* consultanc ${ }^{*}$ OR communication* department* OR communication* advisor* (W1/S1); (ii) organizational communication* OR corporate ${ }^{*}$ communication $^{*}$ OR institutional communication* OR business communication* (W2/S2); (iii) public communication OR government communication $\left(\mathrm{W}_{3} / \mathrm{S}_{3}\right)$. Os operadores booleanos foram usados para inclusão de palavras no singular e no plural (*).

Cada conjunto acima foi relacionado, posteriormente e separadamente, com cada um dos seguintes termos: digital platform*; digital technolog*; information system*. Somadas todas as rodadas de buscas apenas dos termos principais, detectaram-se mais 185 mil artigos. A Tabela 1 consolida os quantitativos de cada conjunto de pesquisa e demonstra um total de 115 selecionados após a exclusão de trabalhos duplicados. Verificase que a base de Web of Science reúne $72 \%$ do número de publicações relacionadas à comunicação e às plataformas digitais. Apenas quatro artigos foram identificados em língua portuguesa, representando os conjuntos W4 e S4. 


\begin{tabular}{lccc}
\hline & $\begin{array}{c}\text { Resultados } \\
\text { termos principais }\end{array}$ & $\begin{array}{c}\text { Após filtro com } \\
\text { termos secundários }\end{array}$ & Selecionados \\
\hline Wo & 63.616 & 5.882 & 9 \\
\hline W1 a W4 & 10.928 & 617 & 91 \\
\hline So & 91.404 & 1.880 & 14 \\
\hline S1 a S4 & 19.794 & 103 & 25 \\
\hline Total & $\mathbf{1 8 5 . 7 4 2}$ & $\mathbf{8 . 4 8 2}$ & $\mathbf{1 3 9}$ \\
\hline & Total selecionados sem duplicados & $\mathbf{1 1 5}$ \\
\hline
\end{tabular}

Tabela 1 - Resultados encontrados nos processos de busca

Embora plataformas digitais sejam ferramentas de trabalho dos departamentos de comunicação, ao analisarmos as duas estratégias de busca se observa que, em ambos os casos, a relação entre plataformas digitais e departamentos de comunicação social representa menos de $1 \%$ dos estudos feitos nos últimos cinco anos. Há diversas lacunas a serem exploradas já os estudos que envolve a comunicação no âmbito digital são recentes. Segundo Garcia-Orosa (2019), a comunicação organizacional online se inicia praticamente ao mesmo tempo que o desenvolvimento da prática profissional. A sistematização de um processo de pesquisa ésempreimportante para manter a continuidadeeo acompanhamento de um determinado tema. No entanto, observa-se que o uso de palavras-chave será sempre um caminho incompleto na identificação de pesquisas ligadas ao objeto de pesquisa de qualquer ordem, pois a diversidade semântica de cada palavra dada a partir experiência de cada pesquisador acaba por levar a rotas que nem sempre se cruzam.

\subsection{Análises global}

Com base nas buscas, foi possível realizar uma análise quantitativa de correlação das palavras-chave e de publicações em periódicos relacionados às áreas de Comunicação e Ciência da Informação. Na Web of Science, identificaram-se quatro artigos (Huertas \& Oliveira, 2019; Rothberg \& de Camargo, 2018; Wolf \& Archer, 2018; Zotova, 2016) a partir da relação entre plataformas ou tecnologias digitais com departamento de comunicação social (W1) e comunicação pública/governamental (W3). Interessante notar que os estudos sobre plataformas digitais não têm se relacionado com aqueles sobre comunicação organizacional (W2), que apresenta o maior quantitativo de artigos identificados. Isso provavelmente é explicado pela forte conexão existente entre os estudos de gestão e de comunicação organizacional com os relacionados à identidade organizacional, cultura organizacional, globalização, poder, liderança e socialização organizacional (Evans, 2016). Apesar da transformação digital das organizações, os pesquisadores de comunicação organizacional ainda precisam explorar as ferramentas digitais e suas interferências nos fluxos de informação e de comunicação. 
Na base de dados Scopus, um artigo (Ao \& Huang, 2020) aparece relacionado a três termos: plataformas digitais (So), comunicação pública/governamental (S1) e comunicação organizacional (S3). Outro artigo selecionado trata dos desafios da informação e comunicação sistematizadas no fortalecimento as organizações públicas (Rangel \& Hernández, 2019), relacionando os conjuntos So e S1.

Uma outra análise visou identificar artigos e revisões ainda mais específicos no que tange a relação entre plataformas digitais e departamentos de comunicação. Para isso, foi coletada uma série de informações, como (a) acesso aberto; origem do periódico, (b) quartis JCR e SJR (c) categoria temática JCR e SJR, (d) Index H SJR, (e) fator de impacto JCR. Com base nesses dados, optou-se por uma nova exclusão de publicações de temáticas relacionadas, por exemplo, a hotelaria, planejamento urbano, geografia, agricultura, saúde, gerontologia, psicologia, engenharia, história e artes, criminologia.

A partir desse recorte, foi possível fazer uma análise mais acurada dos trabalhos publicados que envolvem a temática, notadamente nas áreas de Comunicação, Ciência da Informação e Computação. Embora em 2017 tenha havido um decrescimento; de 2015 ao primeiro quadrimestre de 2020, observa-se aumento das publicações relacionadas à temática comunicação/relações públicas e plataformas digitais (Gráfico 1), totalizando 92 artigos no período.

Todos foram analisados, no entanto esta revisão de literatura teve como foco estudos voltados a uma abordagem mais específica no que se referente a departamentos de comunicação social. Apesar de esse número ser volátil devido à inserção diária de novas publicações nas bases de dados, essa análise dá uma visão geral do aumento de estudos que relacionam tais temáticas.

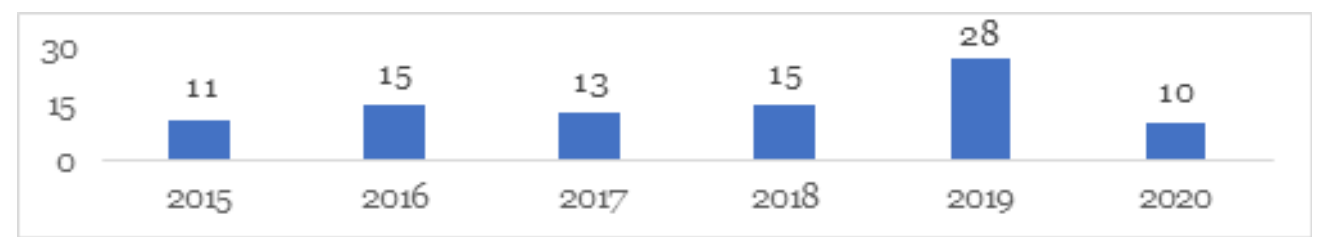

Gráfico 1 - Quantidade de publicações por ano relacionadas à temática comunicação/ relações públicas e plataformas digitais

De 2015 a 2018, houve uma estabilidade no número de publicações. Em 2019, a quantidade quase dobrou em relação ao anterior. Apenas no primeiro quadrimestre de 2020, houve o registro de 10 publicações. Os periódicos Government Information Quarterly, do Reino Unido, e Professional de La Information, da Espanha, registraram quatro publicações, cada um. A revista inglesa possui fator de impacto (2018) de 4.311 e qualificação Q1 - em ambos os indicadores JCR (Biblioteca e Ciência da Informação) e SJR (categoria E-learning, Direito, Biblioteca e Ciência da Informação, Sociologia e Ciência Política). De acesso aberto, o jornal espanhol trata de assuntos ligados à comunicação, informação, indicadores, bibliotecas e tecnologias, sendo qualificado como Q2 (Comunicação, Biblioteca e Ciência da informação), com fator de impacto (2018) 1.505, na base Web of Science; e Q1 (Biblioteca e Ciência da Informação) e 
Q2 (Comunicação e Sistemas de informação) a partir da análise do Scopus. A Public Relations Review (SJR Q1) registrou três artigos publicados.

\subsection{Perspectivas teóricas}

Os dois principais constructos desta revisão sistemática se relacionam em diversos campos de estudos. Ao se trilhar quadros teóricos identificáveis a partir desta metodologia e de outros caminhos já pesquisados, observa-se que as tecnologias digitais passaram pelo determinismo tecnológico, pelo construtivismo social e pela natureza sociotécnica (Ma, 2020). No campo da Ciência da Informação, as abordagens conceituais se encontram associadas à coleta, ao armazenamento e ao uso dos dados. Em estudos de Ciência e Tecnologia, a dedicação está na própria tecnologia, como a coprodução de usuários e de artefatos, a configuração mútua de humanos, do desenho tecnológico e da infraestrutura.

O foco dos pesquisadores de sistema de informação tem sido as plataformas digitais utilizadas nas indústrias, com o intuito de compreender a cadeia de valor e as generalidades voltadas ao lucro (Reuver et al., 2018). No setor público, o núcleo está na capacidade de aprimorar a cocriação de valor a partir da oferta sistemática de produtos e serviços (Hautamäki \& Oksanen, 2018).

Quanto aos estudos em comunicação, há uma preocupação com a democratização das tecnologias digitais para facilitar mudanças sociais positivas, bem como se voltam à lógica que envolve os sistemas digitais. As discussões abrangem a lógica e o controle do poder incorporado em protocolos "que governam a adoção e implementação de tecnologias específicas, e o intercâmbio e o fluxo de dados e informações” (Ma, 2020, p. 147)(MA, 2020).

\subsubsection{O que são plataformas digitais?}

Diante do alerta de Reuver et al. (2018) para que os estudos sempre apresentem o conceito de plataforma digital, evitando assim torná-lo apenas uma moda ou não permitindo a comparabilidade entre pesquisas. Ainda mais ao vê-lo sendo utilizado em diversas ações publicitárias - Youtube, Airbnb, Facebook, Twitter, Globo Play, Disney Play, Amazon Prime. Um dos passos mais importantes nesta investigação foi o de identificar um possível histórico e conceitos.

Verifica-se que, no campo acadêmico, poucas são as discussões conceituais do termo plataforma digital, que teria surgido por volta de 2010, com o seu uso na descrição de serviços online de intermediários de conteúdo para diversos públicos, entre eles consumidores, imprensa e comentaristas (Gillespie, 2010, p. 348).

Em 2014, pesquisadores da Universidade do Porto e de Aveiro, Brasilina, Passarelli, Oliveira e Mealha discutem o termo no livro e-Infocomunicacão - fruto do desenvolvimento do curso doutoral Informação e Comunicação em Plataformas Digitais. Afirmam que o seu uso é visto com frequência em substituição da expressão tecnologia da informação e comunicação. Entendem que por ser digital indica "uma base tecnológica concebida e usada humana e socialmente para que se produza, armazene, recupere, dissemine, comunique e transforme o fluxo informacional" (Passarelli et al., 2014, 1. 2157). 
Quatro anos depois, em a Sociedade da Plataforma, Dijck, Poell e Wall (2018) apresentam os elementos que compõe uma plataforma digital: dados, automatização, organização através de algoritmos e interfaces, formalização através de relações de propriedade impulsionadas por modelos de negócios e regidas por contratos de usuário. Ao se apoiar nesse trabalho, D’Andrea (2020) destaca a capacidade mensurável e de armazenagem das plataformas digitais ao transformar práticas off-line em online, o que fomentaria a ideologia do dataísmo, ou seja, a crença na quantificação objetiva. Não haveria objetividade ou neutralidade ao se escolher o que armazenar e, principalmente, ao interpretar e ao gerir o social a partir de dados estruturados. Ma (2020, p. 145) apresenta o seguinte conceito para tecnologias digitais: "plataformas, caminhos ou canais com características emancipatórias propícias à disseminação de informações, expressão pessoal, deliberação democrática e ação coletiva”.

Todas essas definições são bastantes amplas e servem na prática para descrever sistemas de informação (Marques, 2020), sites, intranets (Rangel \& Hernández, 2019) e notadamente mídias sociais, bem como aplicativos de rede social móvel (Wei, Huang, \& Zheng, 2018). A diferença recai apenas na tela usada, em vez do écran do computador, alguns utilizam predominantemente celulares ou tablets.

A abrangência do seu conceito, no entanto, pode prejudicar a compreensão do seu significado. Com o intuito de dar mais objetividade e clareza, propõe-se a seguinte proposta conceitual para PD: base tecnológica capaz de promover a produção, o armazenamento, a recuperação, a disseminação de informações e a interação, de maneira dialógica ou não dialógica, entre seus usuários.

Vejam que a conceituação de PD aponta assim características relacionadas a sua capacidade de gerir informação como de promover o relacionamento entre pessoas. Após sua conceituação, podemos avançar ao ponto central desta investigação, que envolve as descobertas sobre como os estudos sobre departamentos de comunicação PD se relacionam.

\subsubsection{A transformação digital dos departamentos de comunicação}

Na quarta revolução industrial (Gasca-Hurtado \& Machuca-Villegas, 2019), todas as empresas serão de tecnologia direta ou indiretamente (Walker, Bowkett, \& Duchaine, 2018)disruptive technologies, and the Internet of Things, dependency on in- formation and communication technology (ICT, pois uma empresa não sobrevive longe do uso das tecnologias da informação e comunicação. Com a convergência tecnológica e criação constante de novas formas de comunicação, os departamentos de comunicação social têm sido desafiados a se adaptarem e a criarem novos fluxos de informação e comunicação para atingirem os públicos de interesse.

A implementação de plataformas digitais, nos seus mais diferentes escopos, carrega em seu bojo a redefinição de rotinas organizacionais, culturas, estruturas, habilidades e conhecimentos. Usadas para a mediação infocomunicacional, geram fluxos informacionais estruturados, caracterizados por sua visibilidade e pelo "resultado das atividades e tarefas desenvolvidas de forma repetitiva no ambiente organizacional, apoiados por procedimentos e especificações claras” (Valentim, 2010, pp. 18-19). 
Quando se fala em medição do impacto das TIC, valores de negócio e benefícios tornamse sinônimos, podendo ser classificados como tangíveis (financeiros) e intangíveis ou suaves (não financeiros). Os objetivos de gestão para a implantação de projetos de TIC transitam entre os transacionais, informacionais, estratégicos e infraestruturais (Saleem et al., 2020). Desse modo, o uso de plataformas digitais traz a ideia de uma organização da informação que possa auxiliar na prestação de contas, com o maior controle das práticas de linha de frente, no aumento da vantagem competitiva, no melhor desempenho organizacional e humano.

Nos departamentos de comunicação, a transformação digital ocorre paralelamente com a consolidação da Internet como ferramenta de comunicação e troca de informações, com destaque para o uso constante de redes sociais (Baca et al., 2016; Foroudi \& Montes, 2017; Rincon \& Ortuño, 2018), e de outras ferramentas digitais destinadas ao controle do fluxo informacional e de relacionamento com seus diversos públicos (Androutsopoulou, Karacapilidis, Loukis, \& Charalabidis, 2019).

Diante desse contexto, para avançarmos na discussão, cabe esclarecer o que entendemos como departamento de comunicação social, ou simplesmente, departamento de comunicação. São setores responsáveis por atividades de jornalismo, publicidade e relações públicas em uma organização, seja pública ou privada.

Na América Latina e na Europa, são chamados, comumente, por Secretarias, Departamentos, Diretorias ou Assessorias de Comunicação. Embora alguns estudiosos apontem ser preponderante o rótulo de Relações Públicas, nos Estados Unidos, não há consenso nessa nomenclatura. Muitas universidades têm optado por fundir marketing, comunicação e relações públicas (Rivero Hernández, 2017).

No âmbito da comunicação social, é percebível a atratividade de pesquisadores por temáticas que envolvam estratégias de comunicação ou de marketing voltadas ao fortalecimento da imagem das organizações e seus produtos perante seus públicos-alvo, como Ibrahim, Ismail, Amer, \& Jani (2019). Os pontos centrais das pesquisas tem sido assim estratégias de negócios ou de comunicação - o que Soriano et al. (2018) chamam de práticas de comunicação - a maioria relacionada ao uso de plataformas de mídias sociais, como blogs, Facebook, Twitter e Youtube.

Mais especificamente no caso das relações públicas digitais, há a preponderância de pesquisas voltadas às estratégias de relacionamento entre instituições e seus diversos públicos. Estudos publicados especificamente sobre o tema, entre 2008 e 2014, observaram uma mudança na perspectiva das pesquisas, que passaram da funcional (período de 1960 e 1980) para a co-criacional (a partir de 1990), abrangendo as teorias dialógicas, de excelência, situacional e das partes interessadas. Segundo Huang, Wu, \& Huang (2017), são três os estágios para o desenvolvimento da pesquisa em relações públicas digitais.

O primeiro é chamado de estágio de brotamento (1992-2003), no qual os pesquisadores descrevem a internet de forma geral, sem rigor acadêmico e sem estruturas teóricas, sendo site e e-mail os dois mais frequentes estudos sobre formas de mídia. O segundo é a etapa de diversificação (2004-2007), no qual a internet é estudada como meio de comunicação. É nesse período que são criados os 
blogs (2004) e mídias sociais como o Facebook (2004), Youtube (2006) e o Twitter em 2007. Nesse contexto, as mídias sociais e os sites organizacionais passam a ser os principais objetos de estudos. O terceiro estágio, que se inicia em 2008 e vai até os dias atuais, corresponde a fase de avanço, que examina o potencial das mídias sociais, que passam a alcançar não só pessoas, mas diversas organizações com a criação de suas contas oficiais. Segundo esses autores até 2014, não houve contribuições teóricas radicais.

Em uma realidade de escassez de mão de obra, os profissionais de comunicação acabam por serem multitarefas e incorporando o uso de plataformas digitais como ferramentas de trabalho, sem a capacidade de utilizá-las mais estrategicamente. Embora as plataformas digitais façam parte da rotina desses profissionais, eles as percebem como um instrumental, sem serem capazes de compreender a mudança no paradigma comunicacional, estando imersos no jornalismo analógico, ou seja, a informação segue se difundindo em uma bimedialidade (texto e imagem) e em uma narrativa linear (Baca et al., 2016). São consideradas como parte de um kit de ferramentas de comunicação (Wolf \& Archer, 2018).

Apesar de vistas como ferramentas, os papéis, funções e estruturação organizacional dos departamentos de comunicação passam necessariamente pelo uso das redes sociais. Segundo Rivero Hernández (Rivero Hernández, 2017), seis seriam as áreas de atuação desses departamentos: relações públicas institucionais, mídia, eventos e protocolo; comunicação interna; promoção, divulgação e admissões; mídia (redes internas e sociais); patrocínios, alianças e arrecadação de fundos; e o design do setor, que abrangeria funções para o bom desempenho do departamento. A missão dessas áreas deve abranger o planejamento, o desenvolvimento, o gerenciamento e a supervisão dos processos de comunicação, para o fortalecimento do sentido de pertencimento e fidelização de seus públicos, tornando visível o trabalho institucional, tanto interna quanto externamente. Cabe salientar ainda que, entre as funções desses setores, estão o conhecimento e o atendimento dos cidadãos, jornalistas e cientistas da comunidade, prezando pela disseminação de informações corretas e completas (Salinas, 2016).

O estudo do projeto estrutural de um departamento de comunicação não possui expressividade - o que significa ainda não haver definição de um modelo estrutural dominante. Geralmente a estrutura departamental é baseada no público e no mercado, sendo eficaz aquela capaz de se adaptar a necessidades internas e externas. Essas foram algumas das conclusões do estudo conduzido, em 2017, pela Research Foundation of International Association of Business Communications (IABC). Baseados na Teoria da Excelência em Relações Públicas (Grunig, 2011) e em teorias da administração, Moss, Likely, Sriramesh e Ferrari (2017) entrevistaram chefes de departamentos de comunicação dos cinco continentes: Ásia, África, Europa, América Latina e América do Norte. Entre as principais atividades desses setores, 71,2\% destacaram as atividades baseadas na web. Um dado importante desse estudo é que, das 278 organizações estudadas, 145 (60\%) possuíam até 10 empregados nos departamentos de comunicação, apenas 32 informaram possuir mais de 50.

Alguns estudos têm explorado ligações emergentes entre relações públicas e a gestão de informação, ou seja, o processo sistemático de gerenciamento de dados e informações 
voltado à tomada de decisão. Soriano; Álvarez; Valdés (2018) discutem o termo comunicação estratégica no contexto de alta tecnologia, sendo entendido como o gerenciamento de comunicação entre a organização e seu público, não apresentando diferença substancial no que diz respeito ao próprio conceito de relações públicas apresentado pela Public Relations Society of America (2012).

Ligado ao uso de tecnologias digitais, os pesquisadores apresentam o conceito de Inteligência de Relações Públicas, que expressa a necessidade de conhecimento especializado na coleta de informações para aprimoramento de relacionamentos, "a fim de se adaptar a uma mudança ou ambiente incerto" (Soriano et al., 2018, p. 123). Como informações de valor agregado são a base para a tomada de decisões de qualquer pessoa, setor ou instituição, esse é o modo defendido pelos autores de aumentar a capacidade de uma instituição antecipar, influenciar e colaborar com o sucesso de um negócio.

Também relacionada a essa perspectiva de gerenciamento de dados e informações, Pereira Villazon, Portilla Manjon, \& Rodriguez Salcedo (2019) analisaram 41 trabalhos para investigar o estado da arte relacionado à gestão de Big Data em Relações Públicas. Relacionado ao volume, velocidade e variedade de dados, trazem à discussão conceitos como a veracidade, que representa o grau de confiabilidade das fontes de dados; e o valor, que implica os dados devem ter significado para poder ajudar as organizacionais na tomada de decisão. Reforçam a necessidade de profissionais, como acadêmicos de relações públicas de compreenderem a nova cultura empresarial e econômica na qual a conectividade obriga a redesenhar estratégias de comunicação corporativa.

Pereira Villazon et al. (2019) mostram ainda que um dos principais usos de Big Data se apresenta na comunicação com audiências específicas. Afirmam que o uso de dados e métricas podem ser eficazes para a análise de padrões, como para a análise da probabilidade preditiva para um evento. No entanto, a qualidade de trabalhos que abordam explicitamente a gestão de Big Data em Relações Públicas é escassa. Para eles, se a tendência de os profissionais de relações públicas continuarem a não se darem conta do potencial de gestão de dados, corre-se o risco de ficarem muito atrás de outros campos, ignorando o potencial das contribuições da disciplina de RP no êxito do sistema de Big Data nas organizações.

É preciso que os profissionais de comunicação tenham participação na linguagem e na comunicação decorrente do uso Big Data, contribuindo na construção dos algoritmos e protegendo a esfera social contra práticas discriminatórias. Os algoritmos não são neutros, influenciados pelas escolhas pessoais dos seus autores (Holtzhausen, 2016).

Em países como o Canadá, estudos apontam cinco tecnologias como as principais impulsionadoras da economia: realidade virtual e aumentada; tecnologia móvel de quinta geração; impressão 3D; blockchain, e Inteligência Artificial (Walker et al., 2018). Observa-se assim que o uso de novas tecnologias, de plataformas digitais, tem sido imposto em virtude do processo de transformação digital que atinge toda a sociedade, devendo ser objeto de maior atenção por parte de pesquisadores, profissionais e, principalmente, gestores de comunicação. Embora seja uma temática de difícil acesso por pesquisadores, importante considerar o caminho da evolução histórica de cada país no que se refere ao trabalho e uso dos departamentos de comunicação pelas forças governamentais e políticas (Tantivejakul, 2019). 


\subsubsection{Mídias sociais: a principal perspectiva de estudo de plataformas digitais}

As mídias sociais têm sido vistas como as principais ferramentas de estudo no que se refere a plataformas digitais no âmbito dos estudos de comunicação. De acordo com Kaplan e Haenlein (2010, p. 61), os tomadores de decisão e consultores buscam maneiras pelas quais as empresas podem fazer o uso lucrativo de aplicativos ou mídias sociais, sendo elas consideradas como "um grupo de aplicativos baseados na Internet que se baseiam nas bases ideológicas e tecnológicas da Web 2.0 e que permitem a criação e troca de conteúdo gerado pelo usuário".

As pesquisas de relações públicas têm se concentrado em abordagens relacionais que, segundo Kennedy \& Sommerfeldt (2015), falham na capacidade de demonstrar que as mídias sociais vão além da disseminação de informações, permitindo a ilusão de que promovem diálogo genuíno. Dentro de uma perspectiva pós-moderna, eles defendem que o debate deveria estar sobre o poder presente nas mãos dos profissionais de comunicação decorrente do uso das mídias sociais, o que se deve refletir em estudos sobre ética e obrigações dentro desse sistema de relacionamento. A construção plural do consenso a partir da promoção do dissenso por organizações também poderia estar nas pautas de pesquisas, assim como a compreensão das emoções por meio do jogo de linguagem utilizada que são tidas como irracionais, mas envolvem em si ironia, humor, paródia, sátira e sacarmos. Alertam ainda sobre a necessidade de análise da exclusão digital e da habilidade tecnológica para o uso de mídias sociais com o propósito de fomentá-la em espaços que chamam de subalternos.

Poucos estudos caminham nessa perspectiva pós-moderna. Mosca e Quaranta (2016) tratam da relação entre informação política e participação em sistemas de mídia tradicionais e digitais, entre os quais se destacam petições e boicotes a marcas pelas mídias sociais. Sob a vigência da pandemia da Covid-19, espera-se que emerjam mais pesquisas sobre o papel das mídias sociais na inclusão digital, conforme defende Kennedy \& Sommerfeldt (2015).

Na contramão dessa visão pós-moderna, diversos artigos foram identificados referentes ao uso de mídias sociais na estratégia de comunicação com a população ou com clientes. Allagui e Breslow (2016) investigaram quatro campanhas de relações públicas premiadas que utilizaram, o que eles chamam, de uma variedade de plataformas de mídias sociais. Destacaram que número de curtidas, ROI e o valor equivalente de publicidade têm sido substituídos por conceitos como engajamento, tom, autoridade, atenção, interação, relacionamento e participação.

Ao e Huang (2020) afirmam também que o principal objetivo das pesquisas em termos de tecnologia da informação e comunicação tem sido a medição do engajamento e da capacidade de resposta em sites. Tratam da comunicação dialógica - construção de relacionamentos efetivos entre organizações e públicos por meio da troca de ideias e opiniões - e apresentam os cinco princípios têm sido os mais estudados: loop dialógico, geração de visitas, utilização da informação, conservação dos visitantes e facilidade de interface. Ressaltam ainda a importância do estudo dos antecedentes da implementação do diálogo, como o contexto ambiental, o tipo de comportamento de resposta organizacional, tipo de organização e características, orientação de diálogo online, tipo 
de crise, suporte emocional, competências do profissional, a idade do usuário, o nível de responsabilidade percebido e entendimento de TICs.

Fato é que pesquisadores de diversos países estão estudando as mídias sociais, seja pelo potencial financeiro ou de envolvimento social e político. Na Polônia, pesquisa indicou o aumento da atividade em plataformas de redes sociais como parte da melhoria da governança de comunidades rurais (Krzysztof, 2019). O Twitter foi analisado como ferramenta de comunicação com os cidadãos e melhoria de resultados governamentais em 500 cidades norte-americanas (Feeney, Fusi, Camarena, \& Zhang, 2020). Em Barcelona, Huertas e Oliveira (2019) analisaram mensagens feitas por empresas de turismo pelo Twitter após ataques terroristas à cidade, com o propósito de investigar como esse setor lida com essas situações. É possível identificar ainda estudos sobre aspectos comunicacionais entre consultorias virtuais e seus usuários (Li \& Mao, 2015).

\section{Considerações finais}

Embora a transformação digital tenha chegado aos departamentos de comunicação e seja possível coletar diversos dados produzidos, de modo organizado, falta analisar mais profundamente essa nova realidade. Não há estudos, por exemplo, que explorem o aprimoramento dos processos de trabalho, a otimização dos recursos financeiros e até mesmos tecnológicos.

Uma grande parte dos esforços dos pesquisadores estão voltados a uma melhor compreensão e aprimoramento de relacionamentos com seus diversos públicos, com um olhar especial para as mídias sociais. Sugerem-se estudos que conectem as plataformas digitais aos departamentos de comunicação no sentido de preencher lacunas, principalmente, no que se referente ao poder que os departamentos de comunicação possuem ao serem os responsáveis pelo gerenciamento de diversas PDs de interação com o público-alvo de uma organização.

Apesar de as inovações e transformações decorrentes do uso de tecnologias digitais apontarem pela substituição de diversas atividades do homem pela máquina, os profissionais de comunicação possuem habilidades extremamente humanas que são fundamentais tanto para analisar e dar transparência a uma infinidade de dados, que são produzidos diariamente dentro de uma organização, quanto para contar essa nova parte da história da humanidade.

Assim é fundamental uma perspectiva integradora e holística sobre o papel desses setores nas organizações. É o que se pretende com a próxima etapa do projeto de doutoramento, do que qual esta revisão bibliográfica faz parte. Atenção deve ser dada, principalmente, à lógica de obtenção dos dados baseados no uso das plataformas digitais a partir da perspectiva de profissionais de comunicação. As redes sociais são apenas uma das frentes possíveis a serem exploradas.

\section{Referências}

Allagui, I., \& Breslow, H. (2016). Social media for public relations: Lessons from four effective cases. Public Relations Review, 42(1), 20-30. https://doi.org/10.1016/j. pubrev.2015.12.001 
Androutsopoulou, A., Karacapilidis, N., Loukis, E., \& Charalabidis, Y. (2019). Transforming the communication between citizens and government through AI-guided chatbots. Government Information Quaterly, 36(2), 358-367. https://doi.org/10.1016/j.giq.2018.10.001

Ao, S. H., \& Huang, Q. S. (2020). A systematic review on the application of dialogue in public relations to information communication technology-based platforms: Comparing English and Chinese contexts. Public Relations Review, 46(1). https://doi.org/10.1016/j.pubrev.2019.101814

Baca, A. T., Pascuas, Y. P., Artunduaga, C. R., Ramírez, M. S. P., Palomá, J. S. A., \& Cerón, J. S. S. (2016). Percepciones de los medios periodísticos digitales sobre las tecnologías de la comunicación y la información en el Departamento del Huila (Colombia). Question, 1(49), 515-525.

Cuenca-Fontbona, J., Matilla, K., \& Compte-Pujol, M. (2020). Transformación digital de los departamentos de relaciones públicas y comunicación de una muestra de empresas españolas. Revista de Comunicación, 19(1), 75-92. https://doi.org/10.26441/rc19.1-2020-a5

D’Andréa, C. (2020). Pesquisando Plataformas Online: Conceitos e Métodos. EDUFBA.

Evans, S. K. (2016). Staying Ahead of the Digital Tsunami: The Contributions of an Organizational Communication Approach to Journalism in the Information Age. Journal of Communication, 66(2), 280-298. https://doi.org/10.1111/jcom.12217

Feeney, M. K., Fusi, F., Camarena, L., \& Zhang, F. (2020). Towards more digital cities? Change in technology use and perceptions across small and medium-sized US cities. Local Government Studies, 46(5), 820-845. https://doi.org/10.1080/03003930.2019.1690993

Foroudi, P., \& Montes, E. (2017). Corporate e-communication: Its relationship with corporate logo in the construction of digital interaction platforms. Bottom Line, 3O(3), 201-215. https://doi.org/10.1108/BL-08-2017-0023

Garcia-Orosa, B. (2019). 25 years of research in online organizational communication: Review article. Profesional De La Informacion, 28(5). https://doi.org/10.3145/epi.2019.sep.17

Gasca-Hurtado,G.P.,\&Machuca-Villegas,L.(2019).EradelaCuartaRevoluciónIndustrial. RISTI - Revista Ibérica de Sistemas e Tecnologias de Informação, (34), 11-15. https://doi.org/10.17013/risti.34.0

Grunig, J. E. (2011). Teoria Geral de Excelência em Relações Públicas. In Relações Públicas: teoria, contexto e relacionamento ( $2^{\mathrm{a}}$ ed., pp. 43-129). Difusão Editora.

Hautamäki, A., \& Oksanen, K. (2018). Digital Platforms for Restructuring the Public Sector. In M. L. Smedlund, A. Lindblom (Ed.), Collaborative Value Co-creation in the Platform Economy (pp. 91-108). https://doi.org/10.1007/978-981-10-89565_5 
Holtzhausen, D. (2016). Datafication: threat or opportunity for communication in the public sphere? Journal of Communication Management, 20(1), 21-36. https://doi.org/10.1108/JCOM-12-2014-0082

Huang, Y.-H. C., Wu, F., \& Huang, Q. (2017). Does research on digital public relations indicate a paradigm shift? An analysis and critique of recent trends. Telematics and Informatics, 34(7), 1364-1376. https://doi.org/https://doi.org/10.1016/j. tele.2016.08.012

Huertas, A., \& Oliveira, A. (2019). How tourism deals with terrorism from a public relations perspective: A content analysis of communication by destination management organizations in the aftermath of the 2017 terrorist attacks in Catalonia. Catalan Journal of Communication \& Cultural Studies, 11(1), 39-58.

Ibrahim, I., Ismail, A. F. M. F., Amer, A., \& Jani, S. H. M. (2019). The effectiveness of mass marketing communication as a digital logistics tools in promoting a new online public service platform. International Journal of Supply Chain Management, 8(4), 177-185.

Kaplan, A. M., \& Haenlein, M. (2010). Users of the world, unite! The challenges and opportunities of Social Media. Business Horizons, (53), 59-68. https://doi.org/10.1016/j.bushor.2009.09.003

Kennedy, A. K., \& Sommerfeldt, E. J. (2015). A Postmodern Turn for Social Media Research: Theory and Research Directions for Public Relations Scholarship. Atlantic Journal of Communication, 23(1), 31-45. https://doi.org/10.1080/1545 6870.2015.972406

Krzysztof, K. (2019). Use of information and communication technologies in official websites of Gminas. Are the polish rural and urban-rural local governments prepared to communicate with the younger generation - digital natives, generation Y. Public Administration Issues, (5), 140-154. https://doi.org/10.17323/19995431-2019-0-5-140-154

Li, M., \& Mao, J. (2015). Hedonic or utilitarian? Exploring the impact of communication style alignment on user's perception of virtual health advisory services. International Journal of Information Management, 35(2), 229-243. https://doi.org/10.1016/j. ijinfomgt.2014.12.004

Ma, L. (2020). Rethinking democratizing potential of digital technology: A review of technology and communication studies. Journal of Information, Communication andEthicsinSociety,18(1), 140-156.https://doi.org/10.1108/JICES-02-2019-0022

Marques, B. P. (2020). Aplicações dos sistemas de informação: Quais as áreas de aplicação? Revista Ibérica de Sistemas e Tecnologias de Informação, 4O(12), 11-16. https://doi.org/10.17013/risti.40.0

Moher, D., Liberati, A., Tetzlaff, J., Altman, D. G., Altman, D., Antes, G., ... Tugwell, P. (2009). Preferred reporting items for systematic reviews and meta-analyses: The PRISMA statement. PLoS Medicine. https://doi.org/10.1371/journal. pmed.1000097 
Mosca, L., \& Quaranta, M. (2016). News diets, social media use and non-institutional participation in three communication ecologies: comparing Germany, Italy and the UK. Information Communication \& Society, 19(3), 325-345. https://doi.org/10.1080/1369118X.2015.1105276

Moss, D., Likely, F., Sriramesh, K., \& Ferrari, M. A. (2017). Structure of the public relations/communication department: Key findings from a global study. Public Relations Review, 43(1), 80-90. https://doi.org/10.1016/j.pubrev.2016.10.019

Passarelli, B., Ribeiro, F., Oliveira, L., \& Mealha, O. (2014). Identidade conceitual e cruzamentos disciplinares. In e-Infocomunicação: estratégias e aplicações (pp. 79-121). Senac.

Public Relations Society of America. (2012). Public Relations refined: a modern definition for the new Era of Public Relations. Retrieved January 25, 2021, from http://prdefinition.prsa.org/index.php/2012/04/11/the-modern-definition-ofpublic-relations/\#: :text=Based on a public vote,between organizations and their publics."

Rangel, M. de J. H., \& Hernández, M. L. M. (2019). Desafíos de la información sistematizada y comunicación en el fortalecimiento de organizaciones públicas. Revista de Ciencias Sociales, 25(4), 51-64. https://doi.org/10.31876/rcs. v25i4.30516

Reuver, M. de, Sorensen, C., \& Basole, R. C. (2018). The digital platform: a research agenda. Journal of Information Technology, 33(2), 124-135. https://doi.org/10.1057/s41265016-0033-3

Rincon, A. R. F., \& Ortuño, P. A. H. (2018). Relaciones públicas y sector inmobiliario.: Nuevas estrategias de comunicación digital. Obra Digital: Revista de Comunicación, (15), 31-49. https://doi.org/10.25029/od.2017.176.15

Rivero Hernández, M. (2017). Redimensionamiento del área de Comunicación de la Universidad La Salle Cancún. Revista Internacional de Relaciones Públicas, VII(13), 161-180. https://doi.org/10.5783/revrrpp.v7i13.415

Rothberg, D., \& de Camargo, M. G. (2018). Public Relations and sustainability: the quality of digital public communication of environmental governing organizations. Revista Internacional de Relaciones Publicas, 8(15), 153-176. https://doi.org/10.5783/RIRP-15-2018-09-153-176

Saleem, F., Salim, N., Altalhi, A. H., Ullah, Z., AL-Malaise AL-Ghamdi, A., \& Mahmood Khan, Z. (2020). Assessing the effects of information and communication technologies on organizational development: business values perspectives. Information Technology for Development, 26(1), 54-88. https://doi.org/10.1080/02681102.2017.1335279

Salinas, L. B. (2016). How to analyze the characteristics of communication departments in hospitals and their consequences to citizens? Methodological example. Revista de Comunicacion de La Seeci, 2O(39), 105-126. https://doi.org/http://dx.doi.org/10.15198/seeci.2016.39.105-126 
Soriano, A. S., Alvarez, C. L., \& Valdes, R. M. T. (2018). Bibliometric analysis to identify an emerging research area: Public Relations Intelligence-a challenge to strengthen technological observatories in the network society. Scientometrics, 115(3), 1591-1614. https://doi.org/10.1007/s11192-018-2651-8

Tantivejakul, N. (2019). The never changing story: Eight decades of the government public relations department of Thailand. Public Relations Review, 45(2), 258-266. https://doi.org/https://doi.org/10.1016/j.pubrev.2018.08.009

Valentim, M. L. P. (2010). Ambientes e fluxos de informação. São Paulo: Cultura Acadêmica.

Van Dijck, J., Poell, T., \& De Waal, M. (2018). The platform society: Public values in a connective world. Oxford University Press.

Villazon, T. P., Manjón, I. P., \& Salcedo, N. R. (2019). Big data y Relaciones Públicas. Una revisión bibliográfica del estado de la cuestión. Revista de ComunicacionPeru, 18(1), 151-165. https://doi.org/10.26441/RC18.1-2019-A8

Walker, V., Bowkett, G., \& Duchaine, I. (2018). All Companies Are Technology Companies: Preparing Canadians with the Skills for a Digital Future. Canadian Public Policy, 44(S1), S153-S158. https://doi.org/10.3138/cpp.2018-011

Wei, R., Huang, J., \& Zheng, P. (2018). Use of mobile social apps for public communication in China: Gratifications as antecedents of reposting articles from WeChat public accounts. Mobile Media \& Communication, 6(1), 108-126. https://doi.org/10.1177/2050157917728100

Wolf, K., \& Archer, C. (2018). Public relations at the crossroads: The need to reclaim core public relations competencies in digital communication. Journal of Communication Management, 22(4), 494-509. https://doi.org/10.1108/JCOM-08-2018-0080

Zotova, D. V. (2016). Key Skills of Professionals for Public Relations in Conditions of Modern Communication Systems. S. Voprosy Teorii i Praktiki Zhurnalistiki $=$ Theoretical and Practical Issues of Journalism, 5(1), 164-173. https://doi.org/10.17150/2308-6203.2016.5(1).164-173 